\title{
Uma Revisão Sistemática sobre as contribuições do Design no Ambiente Construído para portadores do Transtorno do Espectro Autista (TEA)
}

\author{
A Systematic Review of how Design contribute to the Built Environment to \\ people with Autistic Spectrum Disorder (ASD)
}

\author{
ALBUQUERQUE, Sheila Rodrigues de \\ Universidade Federal de Pernambuco - UFPE, Mestranda em Design \\ sheilaarq1@gmail.com \\ CORREIA, Walter Franklin Marques \\ PPGDesign-UFPE; PPErgo-UFPE, Dr. Engenharia de Produção \\ wfmc10@gmail.com
}

\begin{abstract}
ARAÚJO, Layane Nascimento de
Universidade Federal de Pernambuco, Mestranda em Design

layane.n.araujo@gmail.com

VILLAROUCO, Vilma

PPGDesign-UFPE; PPErgo-UFPE; PPGAU+D-UFC; Dra. Eng.

vvillarouco@gmail.com
\end{abstract}

\section{RESUMO}

Os espaços físicos podem distrair ou pressionar um usuário portador do Transtorno do Espectro Autista (TEA), pois os mesmos são suscetíveis a estímulos sensoriais. Partindo desse contexto, e com vista nas pesquisas aplicadas ao cenário de ambientes construídos educacionais em sua maioria, entretanto, direcionados aos autistas, o presente estudo teve como objetivo realizar uma revisão sistemática (dos últimos 5 anos). $O$ intuito foi de identificar as contribuições que estão sendo mais relevantes no campo do design e da arquitetura quando se tratam do desenvolvimento de espaços mais adequados, confortáveis e seguros as necessidades dos portadores do TEA. Os resultados encontrados foram claros: diversas pesquisas e recomendações elaboradas por estudiosos mostraram-se significativas com relação às interpretações sensoriais desses usuários com o ambiente em que estão inseridos. 


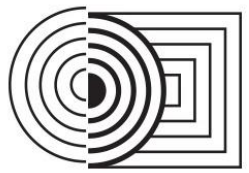

\title{
$17^{\circ}$ ERGODESIGN \& USIHC 2019 \\ PUC-Rio, 11 a 13 de dezembro \\ Rio de Janeiro, RJ, Brasil
}

Palavras-chaves: Autismo, Ambiente Construído, Design, Arquitetura.

\begin{abstract}
Physical spaces can distract or pressure an Autistic Spectrum Disorder (ASD) user because they are susceptible to sensory input. From this context, and with a view to research applied to the scenario of mostly built educational environments, however, directed to the autistic, this study aimed to conduct a systematic review (of the last 5 years). The aim was to identify the contributions that are being most relevant in the field of design and architecture when it comes to developing the most appropriate, comfortable and safe spaces for TEA bearers. The results were clear: several studies and recommendations made by scholars were significant regarding the sensory interpretations of these users with the environment in which they operate.
\end{abstract}

Keywords: Autism, Built environment, Design, Architecture.

\section{INTRODUÇÃO}

\subsection{O Transtorno do Espectro Autista (TEA) e o Ambiente Sensorial}

Segundo o Center for Disease Control and Prevention (CDC), para cada 59 crianças nascidas nos Estados Unidos, 01 é diagnosticada com autismo (BAIO 2018). Sobre isso, Martin (2014), elucida que, o Transtorno do Espectro Autista (TEA) é caracterizado pelo comprometimento de determinadas habilidades, tais como: a comunicação e a interação social, além da presença de comportamentos motores repetitivos. Todavia, Altenmüller-Lewis (2017), explica que, o TEA é considerado como um grupo de deficiências neuro-desenvolvimentais complexas e multifacetadas. No entanto, para os autistas, "a necessidade de estímulos sensoriais nos espaços físicos, os quais podem distrair ou pressionar os usuários, podem promover dificuldades sensoriais como: a hipersensibilidade ou a hipossensibilidade" (DELECATO, 1974).

Baranek et al. (1997) explicam que, os comportamentos estereotipados dos autistas funcionam como mecanismo de defesa sensorial. Entretanto, Baker et al. (2008) afirmam que, esses comportamentos aumentam de acordo com a integração sensorial do autista com ambiente construído.

Por outro lado, Kinnaer et al. (2015), alegam que, os portadores do TEA compreendem o ambiente em que estão inseridos de maneira única e, portanto, as suas experiências e as suas interações são fortemente influenciadas pela suas interpretações espaciais. Sendo assim, Altenmüller-Lewis (2017) ressalta ainda que, para desenvolver ambientes amigáveis e agradáveis para os autistas é importante a compreensão da relação sensorial que os mesmos têm com o ambiente físico.

\subsection{Reflexões a cerca da Arquitetura e do Design para os portadores do TEA}

De acordo com Grandin (1995), o espaço transmite aos autistas uma dimensão simbólica, e assim, estimulando um senso de identidade aos artefatos inerentes nesse espaço, além do favorecimento das interações sociais desses indivíduos com outros usuários do ambiente. 


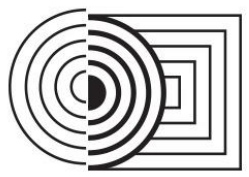

\section{$17^{\circ}$ ERGODESIGN \& USIHC 2019 \\ PUC-Rio, 11 a 13 de dezembro \\ Rio de Janeiro, RJ, Brasil}

$17^{\circ}$ Ergodesign - Congresso Internacional de Ergonomia e Usabilidade de Interfaces Humano Tecnológica: Produto, Informações Ambientes Construídos e Transporte

$17^{\circ}$ USIHC - Congresso Internacional de Ergonomia e Usabilidade

de Interfaces Humano Computador

Em contrapartida, Mostafa (2007) afirma que, o design inclusivo nos espaços físicos foi estudado pelos pesquisadores, porém, inicialmente, tais estudos consideravam mais as questões da acessibilidade física, enquanto que os fatores sensoriais dos ambientes eram menos reconhecidos pelas análises dos pesquisadores.

Por essa razão que, "o ambiente pode ser projetado para alterar favoravelmente as informações sensoriais e assim, criar um espaço mais propício ao desenvolvimento das habilidades e do aprendizado dos autistas" (MOSTAFA, 2008).

Segundo Delmolino e Harris (2012), as estratégias aplicadas aos ambientes escolares para crianças com TEA são muito divergentes. Além disso, os autores criticam também sobre as características ambientais dos projetos em vista do funcionamento dos mesmos, pois no uso de um espaço destinado aos autistas devem-se considerar as características peculiares dos autistas, bem como de todos os envolvidos.

De acordo com Mostafa (2014) a arquitetura é uma ciência capaz de elaborar ambientes, os quais são desenvolvidos em prol das necessidades dos usuários inseridos nos mesmos. No entanto, em vista aos ambientes favoráveis aos usuários autistas, a autora afirma, que as características sobre esse tipo de usuário, geralmente, não são abordadas em códigos e/ou diretrizes de projetos de arquitetura.

Em vista da importância da inserção dos autistas nos espaços educacionais, bem como a prática de políticas públicas que reforçam o direito da inclusão dos portadores do TEA nas escolas, pode-se afirmar, em vista desse contexto e, conforme os estudos de Martin (2014) que, as intervenções espaciais em espaços educacionais ganharam um aumento significativo ao longo dos últimos anos. Todavia, é fundamental que as intervenções relacionadas ao Design sejam coerentes as necessidades dos autistas e como as mesmas podem ser refletidas aos mesmos. É importante compreender também, segundo Kinnaer et al. (2015) que, uma segunda maneira de reorientar o discurso sobre arquitetura favorável para pessoas do TEA é analisando o espaço físico do usuário de dentro para fora e de fora para dentro, porém sob a ótica dos próprios autistas.

De acordo com Love (2018), a versão mais recente publicada pelo Architects and Building Branch (2015) em Londres, destacou a importância em desenvolver espaços mais adequados aos autistas. No entanto, o autor explica que, a versão mais recente ainda contém recomendações voltadas mais para as necessidades especiais gerais. Por outro lado, Barbosa e Fumes (2018) constataram também que, a Norma Brasileira Regulamentadora (NBR 9050/15) não contempla as características dos usuários portadores de transtornos mentais, quando se trata das questões sensoriais.

Portanto, com base nas reflexões realizadas pelos autores apresentados acima, entende-se que, o ambiente físico pode influenciar nas percepções sensoriais dos portadores do TEA. Para tanto, foi visto também que, a arquitetura e o design são ferramentas fundamentais nas intervenções espaciais, e assim, promovendo espaços construídos mais favoráveis às necessidades e ao desenvolvimento dos autistas. Por essa razão que, diretrizes e recomendações são essenciais. Para isso, em vista desse contexto, alguns estudos sobre isso estão apresentados no tópico a seguir.

\subsection{Contribuições da Arquitetura e do Design no Ambiente Construído para os portadores do TEA}




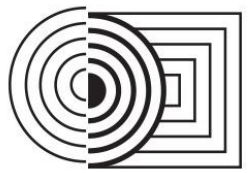

\section{$17^{\circ}$ ERGODESIGN \& USIHC 2019 \\ PUC-Rio, 11 a 13 de dezembro \\ Rio de Janeiro, RJ, Brasil}

$17^{\circ}$ Ergodesign - Congresso Internacional de Ergonomia e Usabilidade de Interfaces Humano Tecnológica: Produto, Informações Ambientes Construídos e Transporte

$17^{\circ}$ USIHC - Congresso Internacional de Ergonomia e Usabilidade de Interfaces Humano Computador

Segundo Vergara \& Rodrigues (2018), os ambientes bem projetados podem proporcionar o aumento da competência dos usuários quando se trata da apropriação do espaço construído. Com vista nessa concepção, compreende-se que, soluções funcionais e eficientes precisam ser elaboradas não apenas a partir das normas e diretrizes, mas principalmente com base nas observações sobre as características e necessidades dos usuários.

As diretrizes e recomendações, discorridas a seguir, são aplicáveis aos ambientes construídos, nos quais venham a ser usufruídos pelos portadores do TEA. Em contrapartida, as propostas podem beneficiar tanto aos usuários atípicos como os indivíduos neurotípicos. Contudo, os seguintes autores recomendam:

- Grandin (1995) destaca a importância do cuidado nas escolhas sobre os materiais e as texturas, devido às chances do sentido tátil dos autistas ser mais apurada do que os demais sentidos. Entretanto, as preferências dos autistas são distintas assim como as suas percepções sensoriais que são únicas;

- Mostafa (2007) explica que, os pequenos espaços íntimos ou espaços de escape são boas soluções para ameninar os baixos e altos estímulos dos autistas;

- "Ladrilhos acústicos, cortinas, pisos resilientes, assentos acolchoados, materiais de teto com absorção de som e distância da iluminação fluorescente" (SCOTT, 2009). São algumas sugestões interessantes para o controle de ruídos e qualidade acústica das salas de aula com alunos portadores do TEA. Além disso, Tufvesson \& Tufvesson (2009) e McAllister (2010) alegam também que, a elaboração de espaços que funcionem como amortecimento de ruídos externos e internos (como corredores) entre as salas de aula também é uma alternativas significativa;

- É fundamental também "a instalação de elementos à prova de som para reduzir ecos e níveis de ruído em espaços onde é desejável um longo tempo de atenção" (MOSTAFA, 2008);

- Mostafa (2010) afirma também que, os locais presentes no ambiente construído devem ser designados de acordo com cada atividade específica. Portanto, o espaço deverá manter um contexto previamente definido, a fim de facilitar a estrutura da rotina do autista, em vista das dificuldades que o mesmo poderá ter ao não identificar um ambiente organizado. Pois, de acordo com Scott (2011), os portadores do TEA são usuários estimulados visualmente. Além disso, os resultados dos estudos realizados por Love (2018), nos testes observacionais, mostraram que, as crianças autistas sentem-se mais confortáveis ao identificar os ambientes de maneira mais organizada e ordenada, de modo que, as funções dos espaços destaquem claramente as suas intenções;

- Brand (2010) recomenda a projetação de ambientes que possam resistir ao uso não intencional, mas que, principalmente sejam duráveis e de fácil manutenção. $O$ autor sugere também que, os ambientes sejam desenvolvidos em vista da proteção e da segurança do indivíduo autista, como por exemplo, o uso de superfícies maleáveis. Pois, esses usuários podem apresentar um comportamento chamado: Resposta ao acerto, que é caracterizado a qualquer tipo de acerto, e isto inclui: crianças batendo em si mesmas ou jogando objetos que podem ferir a si mesma ou a outros. 
- Grandin (2012) elucida que, o Design da Sala de Aula deve proporcionar conforto e apoio as crianças com TEA, e para isso, o autor recomenda as terapias sensoriais como método de alívio emocional, e desta maneira, facilitando o desenvolvimento das habilidades das crianças. Tais terapias sensoriais podem ser refletidas a partir da projetação de mobiliário e de equipamentos especializados para o ambiente construído utilizado por esses indivíduos;

- Outras recomendações, com base nas pesquisas de Kanakri et al. (2016) podem ser consideradas para a melhoria acústica dos ambientes como: o uso de um sistema AVAC (Aquecimento, Ventilação e Ar Condicionado) melhor; alternativas para regulação do ar; revestimentos para a absorção de ruídos nas paredes; carpetes e outros materiais absorventes de som no chão; um buffer entre salas de aula e a atenção aos espaços externos; além da análise para saber se o acesso às vistas naturais melhora o bemestar ou aumenta os problemas acústicos, bem como também o controle dos índices de ruídos, os quais devem ser de aproximadamente $50 \mathrm{~dB}$ ou menos.

Isto, porque, segundo os estudos de Howe e Stagg (2016), portadores do TEA sob os efeitos desagradáveis do ruído podem apresentar: frustração, aborrecimento e desconforto físico como comportamentos mais frequentes, quando presentes em ambientes com problemas acústicos. Além disso, os autores mostraram em seus estudos recentes que $88 \%$ das crianças com autismo apresentam algum desconforto relacionado à acústica quando estão inseridos dentro do ambiente escolar.

- Entretanto, Matin et al. (2017) comprovaram em seus estudos que o uso de bolas terapêuticas como assento ajudam no controle dos estímulos dos autistas.

- De acordo com Martin (2014), os Designers, podem promover soluções ou modificações nos ambientes construídos, a partir de observações relacionadas às condições fisiológicas, sociais, psicológicas dos autistas. Para isto, McAllister e Maguire (2012), reforçam também que, os arquitetos podem proporcionar ambientes internos e externos eficientes, confortáveis e seguros a partir da concepção de projetos que visem às condições fundamentais dos portadores do TEA.

Com base no contexto discorrido acima, é notório que, diversos estudos já foram concebidos em vista de projetos ambientais para os usuários autistas. Entretanto, as pesquisas sobre esse cenário e a promoção dessas diretrizes e estudos mais aprofundados ainda precisam ser mais reconhecidos e tratados com mais ênfase pelas normas regulamentadoras, assim como pelos projetistas.

A OMS (2016) também reforça que, os arquitetos devem projetar ambientes adequados e eficientes quanto às atividades que serão desenvolvidas nos mesmos. No entanto, foi visto também que, para projetar ambientes construídos mais inclusos e mais coerentes às condições e às necessidades dos usuários, os profissionais devem partir de um fator chave indispensável: a observação quanto às interpretações sobre as percepções sensoriais dos autistas. 


\section{METODOLOGIA}

Foi realizada para este estudo, uma Revisão Sistemática da Literatura (RSL), iniciada em março de 2019, tendo em vista a análise de pesquisas nacionais e internacionais. Para isso, na primeira etapa, a revisão sistemática ocorreu a partir dos seguintes procedimentos: a Base de Dados foi o Portal de Periódicos CAPES, e a Busca Avançada foi escolhida como: "Qualquer", ou seja, os conteúdos foram localizados pelo título, ou pelo autor, ou pelo assunto.

Os critérios de inclusão foram: somente artigos, revisados por pares, período: últimos 5 anos (2014-2019), título com foco nas palavras-chaves, e os 1.000 primeiros listados (para resultados $>5000$ ), e os 100 primeiros listados (para resultados $<5000$ ) e conteúdos em qualquer idioma.

Todavia, para os critérios de exclusão foram: por título (sem foco com as palavras-chaves) e artigos repetidos. Para tanto, 8 combinações (quatro em inglês e quatro em português) com as palavras-chaves foram realizadas nesta RSL (ver Tabela 01).

Tabela 1. Revisão Sistemática

\begin{tabular}{|c|c|c|c|c|c|c|}
\hline $\begin{array}{l}\text { Palavra- } \\
\text { Chave }\end{array}$ & $\begin{array}{l}\text { Operador } \\
\text { Booleano }\end{array}$ & Palavra-Chave & $\begin{array}{c}\text { Total de artigos } \\
\text { publicados }\end{array}$ & $\begin{array}{c}\text { Total de } \\
\text { artigos } \\
\text { visualizados }\end{array}$ & $\begin{array}{c}\text { Artigos } \\
\text { selecionado } \\
\text { s pelo título }\end{array}$ & $\begin{array}{c}\text { Artigos } \\
\text { relacionados } \\
\text { ao tema da } R S\end{array}$ \\
\hline Autismo & AND & $\begin{array}{l}\text { Ambiente } \\
\text { Construído }\end{array}$ & 20 & \multirow{9}{*}{1224} & 0 & 0 \\
\hline Autismo & AND & Salas de Aula & 10 & & 2 & 0 \\
\hline Autismo & AND & Ergonomia & 1 & & 0 & 0 \\
\hline Autismo & AND & Acessibilidade & 13 & & 3 & 3 \\
\hline Autism & AND & $\begin{array}{c}\text { Environment } \\
\text { Built }\end{array}$ & 15 & & 3 & 0 \\
\hline Autism & AND & Classroom & $5297^{*}$ & & 35 & 3 \\
\hline Autism & AND & Ergonomic & 65 & & 3 & 3 \\
\hline Autism & AND & Design & $28122^{*}$ & & 1 & 0 \\
\hline \multicolumn{3}{|c|}{ TOTAL } & 33543 & & 47 & 9 \\
\hline
\end{tabular}

Fonte: realizado pelos autores (2019)

Além disso, dos 5297 artigos encontrados nos resultados (da combinação: Autism AND Classroom), apenas os 1000 primeiros artigos (por ordem de relevância) que foram visualizados pelo título. E dos 28122 artigos encontrados nos resultados (da combinação: Autism AND Design), apenas os 100 primeiros artigos (por ordem dos mais acessados) que foram visualizados pelo título. Portanto, ao todo foram 1224 artigos visualizados.

\section{RESULTADOS}

Nove artigos foram selecionados e analisados para o desenvolvimento desta Revisão Sistemática, 6 artigos internacionais e 3 artigos nacionais, nos quais estão descritos a seguir: 
$17^{\circ}$ ERGODESIGN \& USIHC 2019

PUC-Rio, 11 a 13 de dezembro

Rio de Janeiro, RJ, Brasil $17^{\circ}$ Ergodesign - Congresso Internacional de Ergonomia e Usabilidade de Interfaces Humano Tecnológica: Produto, Informações Ambientes Construídos e Transporte

$17^{\circ}$ USIHC - Congresso Internacional de Ergonomia e Usabilidade de Interfaces Humano Computador

Quadro 1. Sistematização do Primeiro Artigo da Revisão Sistemática

\begin{tabular}{|c|c|}
\hline Ano & 2014 \\
\hline Título & Architecture For Autism: Autism Aspects in School Design. \\
\hline Autor & MOSTAFA, M. \\
\hline Objetivo & $\begin{array}{l}\text { llustração da aplicação dos princípios das diretrizes de design em um Centro de } \\
\text { Educação Avançada. }\end{array}$ \\
\hline Métodos & Modelo da Teoria do Design Sensorial. \\
\hline Resultados & O impacto significativo do design para autistas nos ambientes educacionais. \\
\hline Publicação & International Journal of Architectural Research. \\
\hline Local & Índia. \\
\hline
\end{tabular}

Fonte: realizado pelos autores (2019)

O primeiro artigo analisado nesta Revisão Sistemática é referente a uma investigação realizada por Mostafa (2014), na qual realizou diversos estudos que resultaram em recomendações significativas para o campo da arquitetura e do design, e que podem ser implantadas em projetos de escolas que desejam tornar os seus ambientes mais inclusivos para as necessidades dos usuários autistas.

A autora seguiu com base no modelo da Teoria do Design Sensorial, uma ferramenta flexível e adaptável, a qual tem por objetivo fornecer diretrizes para um projeto de arquitetura que visa 0 foco nos estímulos sensoriais dos ambientes, e assim, reforçando a concepção de que, a mudança no ambiente sensorial poderá trazer impactos diretos nos comportamentos estereotipados dos autistas. Sendo assim, as sugestões da autora resumem-se nas seguintes propostas:

Do ponto de vista micro de um espaço, considerando o ambiente interior, intervenções aplicadas à acústica, inserção de uma sequência espacial, espaços de escape, propostas voltada à segurança, compartimentalização, opções de zonas de transição e zoneamento sensorial são algumas das estratégias que podem transformar o ambiente micro (interno) mais adequado e confortável aos autistas. No entanto, a autora destaca também, outros pontos importantes a serem considerados quando se trata do ambiente escolar.

Dessa forma, a autora propõe que, o design geral da sala de aula, assim como espaços especializados a terapia e locais de aprendizagem ao ar livre devem ser reconhecidos nesses ambientes. Por fim, a autora esclarece que, as alternativas sob a ótica do contexto do entorno e da comunidade, bem como as questões voltadas a navegação, a circulação e a sequência 


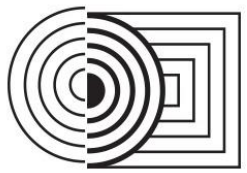

$17^{\circ}$ ERGODESIGN \& USIHC 2019

PUC-Rio, 11 a 13 de dezembro

Rio de Janeiro, RJ, Brasil $17^{\circ}$ Ergodesign - Congresso Internacional de Ergonomia e Usabilidade de Interfaces Humano Tecnológica: Produto, Informações Ambientes Construídos e Transporte

$17^{\circ}$ USIHC - Congresso Internacional de Ergonomia e Usabilidade de Interfaces Humano Computador

espacial são estratégias fundamentais para os autistas que também usufruem dos espaços externos.

Quadro 2. Sistematização do Segundo Artigo da Revisão Sistemática

\begin{tabular}{|c|c|}
\hline Ano & 2015 \\
\hline Título & $\begin{array}{r}\text { Autism-Friendly Architecture From The Outside In And The Inside Out: An } \\
\text { Explorative Study Based On Autobiographies Of Autistic People }\end{array}$ \\
\hline Autor & KINNAER, M.; BAUMERSI, S.; HEYLIGHEN, A. \\
\hline Objetivo & $\begin{array}{c}\text { Realizar a identificação dos usuários do TEA, a importância do ambiente } \\
\text { construido, a sua interpretação e a maneira correspondente de lidar com ele, } \\
\text { porém, sob dois pontos de vista espacial. }\end{array}$ \\
\hline $\begin{array}{c}\text { Métodos } \\
\text { Estudo bibliográfico de } 6 \text { autobiografias de autistas e em paralelo o confronto } \\
\text { com diretrizes do Guia de Design. }\end{array}$ \\
$\begin{array}{c}\text { O ambiente construido pode promover possibilidades de uso e integração do } \\
\text { usuário e tornando-se tão importante quanto às próprias diretrizes de design. }\end{array}$ \\
Lublicação
\end{tabular}

Fonte: realizado pelos autores (2019)

O segundo artigo avaliado, abordou sobre reflexões a cerca do desenvolvimento de ambientes adequados aos autistas, porém, considerando o relato de interpretações espaciais de portadores do TEA e em paralelo a análise de determinados elementos essenciais para um projeto mais agradável, confortável e seguro a estes usuários.

Os elementos selecionados para os estudos desse artigo foram: o Guia de Design, uma seleção de autobiografias de autistas. Os autores seguiram as suas análises a partir de dois pontos de vista espacial: do exterior para o interior e do interior para o exterior.

O primeiro ponto de vista (do exterior para o interior) aborda quatro temas essenciais para a exploração do ambiente construído em vista das perspectivas dos usuários autistas. O segundo tema trata sobre as questões acústicas em um ambiente construído. O terceiro tema foi estudado com base no ponto de vista da escala urbana e o quarto tema abordado no ponto de vista espacial por Kinnaer et al. (2015) caracterizou pelas condições da estrutura física dos ambientes. O segundo ponto de vista, parte da investigação da perspectiva do interior para o exterior do ambiente construído e foi visto que, os autistas constroem uma identidade de continuidade com ambiente material.

Para tanto, foi visto que, as autobiografias dos autistas mostraram que, o ambiente construído pode promover possibilidades de uso e integração do usuário e tornando-se tão importante quanto às próprias diretrizes de design. Pois, cada usuário autista estrutura e relaciona a sua 
$17^{\circ}$ ERGODESIGN \& USIHC 2019

PUC-Rio, 11 a 13 de dezembro

Rio de Janeiro, RJ, Brasil $17^{\circ}$ Ergodesign - Congresso Internacional de Ergonomia e Usabilidade de Interfaces Humano Tecnológica: Produto, Informações Ambientes Construídos e Transporte

$17^{\circ}$ USIHC - Congresso Internacional de Ergonomia e Usabilidade de Interfaces Humano Computador

realidade de acordo com a sua forma peculiar de interpretação do espaço. No entanto, as concepções extraídas pelos pesquisadores concluíram que, as diretrizes de design devem promover neutralidade e equilíbrio adequado às necessidades dos indivíduos.

Quadro 3. Sistematização do Terceiro Artigo da Revisão Sistemática

\begin{tabular}{|c|c|}
\hline Ano & 2017 \\
\hline Título & Designing Schools for Students on the Spectrum \\
\hline Autor & ALTENMÜLLER-LEWIS, U. \\
\hline Objetivo & $\begin{array}{c}\text { Analisar diretrizes para projetos de ambientes de aprendizagem, em vista do } \\
\text { desenvolvimento social e intelectual dos portadores do TEA. }\end{array}$ \\
\hline Métodos & Estudo bibliográfico sobre a Matriz de Design Sensorial. \\
\hline Resultados & $\begin{array}{l}\text { Apresentação de } 11 \text { diretrizes que podem ser aplicadas a projetos } \\
\text { arquitetônicos, os quais buscam atender as necessidades dos autistas. }\end{array}$ \\
\hline Publicação & Sapienza University of Rome. \\
\hline Local & Itália \\
\hline
\end{tabular}

Fonte: realizado pelos autores (2019)

O terceiro artigo destaca uma análise da autora sobre os estudos feitos por Mostafa (2014), com foco na abordagem sensorial dos autistas. Pois, o mau funcionamento desse ambiente pode resultar nos autistas aumentos nos comportamentos estereotipados. Por esse motivo que, a autora baseou os seus estudos a partir da Matriz de Design Sensorial, na qual funciona através do rastreamento de impulsos sensoriais dentro do ambiente construído.

Com base nesse contexto, a autora descreveu 11 diretrizes que podem aplicadas em projetos de ambientes de aprendizagem, em vista do desenvolvimento social e intelectual dos portadores do TEA. Dessa forma, as diretrizes resumem-se aos seguintes aspectos: Segurança, Contexto e Comunidade; Zoneamento e Compartimentalização; Sequenciamento Espacial; Limites; Localização, Navegação e Circulação; Espaços de Escape e Quartos Sensoriais; Controle de Estímulos Sensoriais; Acústica; lluminação e Cor.

Em suma, Altenmüller-Lewis (2017), apresentou recomendações específicas para dar suporte aos profissionais que pretendem tornar o projeto mais adequado às necessidades gerais dos autistas. E que, além disso, o perfil comportamental e sensorial de cada autista é distinto e singular, e que isto deve ser assimilado durante a concepção de um projeto. 
$17^{\circ}$ ERGODESIGN \& USIHC 2019

PUC-Rio, 11 a 13 de dezembro

Rio de Janeiro, RJ, Brasil $17^{\circ}$ Ergodesign - Congresso Internacional de Ergonomia e Usabilidade de Interfaces Humano Tecnológica: Produto, Informações Ambientes Construídos e Transporte

$17^{\circ}$ USIHC - Congresso Internacional de Ergonomia e Usabilidade de Interfaces Humano Computador

Quadro 4. Sistematização do Quarto Artigo da Revisão Sistemática

\begin{tabular}{|c|c|}
\hline Ano & 2014 \\
\hline Título & $\begin{array}{l}\text { Exploring the impact of the design of the physical classroom environment on } \\
\text { young children with autism spectrum disorder (ASD). }\end{array}$ \\
\hline Autor & MARTIN, C. S \\
\hline Objetivo & $\begin{array}{l}\text { Aprofundar os conhecimentos sobre o Design em espaços físicos educacionais } \\
\text { em vista dos autistas, no intuito de servir como orientação para os projetistas. }\end{array}$ \\
\hline Métodos & $\begin{array}{l}\text { Revisão Sistemática da Literatura (RSL) sobre estudos aplicados ao ambiente } \\
\text { físico para autistas. }\end{array}$ \\
\hline Resultados & $\begin{array}{l}\text { A RSL apresentou orientações significativas para projetos de ambientes } \\
\text { educacionais, em vista dos usuários autistas. No entanto, as pesquisas sobre } \\
\text { essa temática ainda precisam ser mais exploradas. }\end{array}$ \\
\hline Publicação & Journal of Research in Special Educational Needs, Inglaterra \\
\hline Local & Inglaterra \\
\hline
\end{tabular}

Fonte: realizado pelos autores (2019)

$\mathrm{Na}$ análise do quarto artigo foi visto que, Martin (2014) realizou uma Revisão Sistemática da Literatura (RSL), onde dos 23 artigos analisados em sua pesquisa, apenas 13 trabalhos, apresentaram estudos aplicados ao ambiente físico para autistas, e os demais se concentraram apenas nas necessidades educacionais dos portadores do TEA.

Conforme a autora, o National Standards Project recomenda que, a elaboração de intervenções seja realizada a partir da análise de evidências, ou seja, a partir de observações de casos empíricos. Para que dessa forma, promova a eficiência do projeto aplicado no determinado ambiente estudado.

A pesquisa também foi desenvolvida em virtude da observação do aumento das intervenções em espaços educacionais para autistas. Pois os estudos mostraram a importância das orientações e das recomendações em vista do contexto do design aplicado ao ambiente educacional.

Portanto, a autora elucida a importância da influência do design no ambiente, assim como a exploração de mais pesquisas relacionadas ao usuário (autista) em função do espaço, e reflete também sobre os métodos aplicados na investigação dos ambientes pelos profissionais das áreas de psicologia, educação e design. 
$17^{\circ}$ ERGODESIGN \& USIHC 2019

PUC-Rio, 11 a 13 de dezembro

Rio de Janeiro, RJ, Brasil $17^{\circ}$ Ergodesign - Congresso Internacional de Ergonomia e Usabilidade de Interfaces Humano Tecnológica: Produto, Informações Ambientes Construídos e Transporte

$17^{\circ}$ USIHC - Congresso Internacional de Ergonomia e Usabilidade de Interfaces Humano Computador

Quadro 5. Sistematização do Quinto Artigo da Revisão Sistemática

\begin{tabular}{|c|c|}
\hline Ano & 2018 \\
\hline Título & $\begin{array}{c}\text { Sensory Spaces: Sensory Learning - An Experimental Approach To Educating } \\
\text { Future Designers To Design Autism Schools. }\end{array}$ \\
\hline Autor & LOVE, J. \\
\hline Objetivo & $\begin{array}{l}\text { Desenvolver sugestões e reconhecer as necessidades de um projeto } \\
\text { arquitetônico educacional direcionado para usuários autistas. }\end{array}$ \\
\hline Métodos & Estudo experimental, Pesquisa Bibliográfica e Trabalhos em Grupo Online. \\
\hline Resultados & $\begin{array}{l}\text { Os estudantes de Design identificaram a importância do aprofundamento das } \\
\text { pesquisas devido a elaboração de recomendações significativas e coerentes às } \\
\text { necessidades dos autistas. }\end{array}$ \\
\hline Publicação & International Journal of Architectural Research. \\
\hline Local & Reino Unido. \\
\hline
\end{tabular}

Fonte: realizado pelos autores (2019)

No quinto artigo analisado neste trabalho foi baseado em um estudo de um projeto experimental realizado por quatro estudantes de design. De acordo com o autor, o objetivo do projeto abordado é de reconhecer o projeto de escolas direcionadas para usuários autistas de 11 a 19 anos.

O intuito do estudo consistiu na participação direta dos autistas e dos estudantes. O projeto foi acompanhado por dois professores de design, um com experiência numa escola de artes e com autistas, e outro com experiência em escolas de arquitetura. $O$ trabalho apresentado pelo autor foi dividido nas seguintes partes: Parte I: Escopo do projeto. Parte II: Participação direta dos autistas. Parte III: Apresentação dos resultados. Parte IV: Conclusões.

Os estudantes de design realizam algumas técnicas para aprimorar os conhecimentos sobre tema proposto no projeto, os quais foram: briefing, análise do filme: Thinking in Pictures, revisão bibliográfica, estudos aprofundados sobre o Autismo e um Trabalho em Grupo online.

Entretanto, alguns requisitos foram determinados para a concepção do projeto, tais como: clareza na organização do ambiente (a fim de se evitar funções mistas ou confusas no espaço), baixo estímulo no ambiente (cores, iluminação, texturas, etc. adequadas). O projeto exigiu também que, o trabalho considerasse as crianças autistas verbais, não verbais, com grau severo e as de alto funcionamento.

$\mathrm{Na}$ última parte do projeto foi possível compreender que o projeto de design recebeu uma importante integração dos estudantes, bem como as pesquisas realizadas sobre o autismo, as quais contribuíram com a concepção do projeto. 
$17^{\circ}$ ERGODESIGN \& USIHC 2019

PUC-Rio, 11 a 13 de dezembro

Rio de Janeiro, RJ, Brasil $17^{\circ}$ Ergodesign - Congresso Internacional de Ergonomia e Usabilidade de Interfaces Humano Tecnológica: Produto, Informações Ambientes Construídos e Transporte

$17^{\circ}$ USIHC - Congresso Internacional de Ergonomia e Usabilidade de Interfaces Humano Computador

Quadro 6. Sistematização do Sexto Artigo da Revisão Sistemática

\begin{tabular}{|c|c|}
\hline Ano & 2016 \\
\hline Título & $\begin{array}{c}\text { An Observational Study of Classroom Acoustical Design and Repetitive Behaviors in Children } \\
\text { With Autism. }\end{array}$ \\
\hline Autor & KANAKRI, S. M.; SHEPLEY, M.; TASSINARY, L. G.; VARNI, J. W.; FAWAZ, H. M. \\
\hline Objetivo & $\begin{array}{l}\text { A pesquisa buscou analisar as relações entre os comportamentos repetitivos e o } \\
\text { processamento auditivo, bem como a verificação dos comportamentos em vista dos níveis de } \\
\text { ruído em um ambiente controlado. Além de recomendações a acústica de ambientes de sala de } \\
\text { aula. }\end{array}$ \\
\hline Métodos & Estudos de casos comparativos; Revisão Bibliográfica e o uso do Programa Noldus XT. \\
\hline Resultados & $\begin{array}{c}\text { Os autores comprovaram que, tanto nos ambientes silenciosos quanto nos barulhentos foram } \\
\text { apresentados pelos autistas comportamentos estereotipados. }\end{array}$ \\
\hline Publicação & Journal Environment and Behavior. \\
\hline Local & Reino Unido. \\
\hline
\end{tabular}

Fonte: realizado pelos autores (2019)

O trabalho desenvolvido por Kanakri et al. (2016) consistiu no levantamento bibliográfico sobre a relações entre: o Ruído e o Ambiente construído; o Autismo e o ruído na sala de aula e o Autismo relacionado com o ambiente construído.

O objetivo geral da pesquisa dos autores baseou-se em testes com relação à prevalência de comportamentos repetitivos em análise do aumento dos níveis de ruído. Desse modo, compreender como as alterações acústicas no ambiente construído podem impactar no aprendizado e beneficiar no desenvolvimento das crianças com autismo.

Os estudos de casos experimentais foram realizados com duas escola durante 7 semanas. Ao todo foram 20 crianças (16 meninos e 04 meninas) com autismo com alto funcionamento, e que estudavam na segunda e na terceira série, numa escola específica para autistas.

Duas salas de aula (uma barulhenta e outra silenciosa) com crianças de cada escola participaram do estudo. Os materiais usados pelos pesquisadores foram: um gravador de vídeo e um decibelímetro. $\mathrm{Na}$ análise da confiabilidade dos dados, os pesquisadores utilizaram o programa Noldus XT.

Os resultados mostraram que, tanto nos ambientes silenciosos quanto nos barulhentos foram apresentados pelos autistas comportamentos estereotipados, tais como: movimento motor repetitivo, seguido pela fala repetitiva, emissão de sons altos, cobrindo os ouvidos, piscando os olhos, reclamando e batendo nos outros.

Por fim, os autores compreenderam a importância da acústica e o controle dos ruídos nos comportamentos dos autistas, e que isto é um fator fundamental, o qual precisa ser considerado nos projetos arquitetônicos. 
$17^{\circ}$ ERGODESIGN \& USIHC 2019

PUC-Rio, 11 a 13 de dezembro

Rio de Janeiro, RJ, Brasil $17^{\circ}$ Ergodesign - Congresso Internacional de Ergonomia e Usabilidade de Interfaces Humano Tecnológica: Produto, Informações Ambientes Construídos e Transporte

$17^{\circ}$ USIHC - Congresso Internacional de Ergonomia e Usabilidade de Interfaces Humano Computador

Quadro 7. Sistematização do Sétimo Artigo da Revisão Sistemática

\begin{tabular}{|c|c|}
\hline Ano & 2018 \\
\hline Titulo & Acessibilidade entre Mundos: uma Arquitetura mais Inclusiva aos Autistas. \\
\hline Autor & VERGARA, L. G. L. TRONCOSO, M. U. RODRIGUES, G. V. \\
\hline Objetivo & $\begin{array}{l}\text { Análise de diretrizes de arquitetura para a proposição de soluções projetuais a ambientes } \\
\text { escolares. }\end{array}$ \\
\hline Métodos & Pesquisa Bibliográfica. \\
\hline Resultados & $\begin{array}{l}\text { As autoras destacaram diretrizes que promovem a qualidade de vida dos portadores do TEA, } \\
\text { em análise a recomendações ambientais inseridas no contexto das interações do social: } \\
\text { imediato, próximo e sociocultural. }\end{array}$ \\
\hline Publicação & ENEAC. \\
\hline Local & Brasil. \\
\hline
\end{tabular}

Fonte: realizado pelos autores (2019)

As autoras buscam refletir, neste trabalho, que não somente as terapias contribuem para essa evolução e sim, a inserção de uma arquitetura adequada às características dos portadores do TEA.

Do ponto de vista social, as mesmas apontam que, as soluções designadas ao ambiente construído dos autistas devem considerar três tipos de interações sociais essenciais a vida de um indivíduo autista e que, portanto, são: o social imediato (onde inclui o sujeito e seus familiares), o social próximo (que são os vizinhos, a comunidade e a escola) e o sociocultural (que diz respeito à população em geral, em vista da acessibilidade universal).

Em suma a esta reflexão, as autoras concluíram que, o ambiente construído é um conjunto de características construtivas e físicas, as quais são indissociáveis com relação ao ambiente social. E que, além disso, o planejamento das cidades também deve ser reconhecido para os portadores do TEA, pois para as autoras, esse contexto já vem sendo observado há décadas por importantes críticos do urbanismo moderno. 
$17^{\circ}$ ERGODESIGN \& USIHC 2019

PUC-Rio, 11 a 13 de dezembro

Rio de Janeiro, RJ, Brasil $17^{\circ}$ Ergodesign - Congresso Internacional de Ergonomia e Usabilidade de Interfaces Humano Tecnológica: Produto, Informações Ambientes Construídos e Transporte

$17^{\circ}$ USIHC - Congresso Internacional de Ergonomia e Usabilidade de Interfaces Humano Computador

Quadro 8. Sistematização do Oitavo Artigo da Revisão Sistemática

\begin{tabular}{|c|c|}
\hline Ano & 2018 \\
\hline Título & A Ergonomia e a Arquitetura no Cuidado das Pessoas com Transtorno Mental. \\
\hline Autor & VASCONCELOS, M. C.; FALCÃO, C. S. \\
\hline Objetivo & $\begin{array}{l}\text { Discussão de diretrizes para uma adequada análise das condições ambientais } \\
\text { e proposta de adaptação conforme as características levantadas. }\end{array}$ \\
\hline Métodos & $\begin{array}{l}\text { Pesquisa Bibliográfica e Elaboração de Mapa de Riscos com base na NR } 9 \\
\text { (1978). }\end{array}$ \\
\hline Resultados & $\begin{array}{l}\text { A partir da realização dos estudos de caso, as autoras elaboraram } \\
\text { recomendações que poderão ser aplicadas em projetos de ambientes } \\
\text { construidos para os portadores do TEA. }\end{array}$ \\
\hline Publicação & ENEAC \\
\hline Local & Brasil \\
\hline
\end{tabular}

Fonte: realizado pelos autores (2019)

Nesta oitava análise da RS foi realizado pelas autoras um mapeamento das dificuldades nas atividades diárias em seus respectivos ambientes, apontando os riscos e indicando os usos que a pessoa com transtorno faz em cada ambiente.

De acordo com Vasconcelos e Falcão (2018), as pessoas com transtornos mentais costumam sair de seus ambientes particulares para realizar suas tarefas cotidianas com o mundo exterior. No entanto, quando se trata do ambiente próprio à atenção deve ser um fator a ser considerado com relação aos cuidados especiais. A fim de oferecer suporte ao ambiente residencial dos portadores do TEA. As autoras afirmam que, quando ocorre a presença de um diagnóstico de um autista numa família, uma séria de mudanças aparece no convívio familiar como: cuidados especiais da saúde daquela pessoa, tratamentos medicamentosos, terapias, atenção diferenciada à sua higiene, alimentação e cognição.

E por esse motivo, as mesmas alegam que, a arquitetura de sua residência nem sempre é considerada, e por esse fato, problemas nas atividades do cotidiano podem afetar 0 desenvolvimento das mesmas sejam elas no ambiente interno ou externo dos portadores do TEA. Por fim, as análises das autoras, consistiram na identificação das características das atividades, e assim, permitiu que as diretrizes do projeto, baseadas no Mapa de Riscos orientado pela NR 9 fossem traçadas, no intuito de promover a saúde, o conforto e a segurança do autista e da sua família. E desse modo, minimizando os riscos encontrados e consequentemente melhorando a qualidade de vida dos usuários envolvidos. 
$17^{\circ}$ ERGODESIGN \& USIHC 2019

PUC-Rio, 11 a 13 de dezembro

Rio de Janeiro, RJ, Brasil $17^{\circ}$ Ergodesign - Congresso Internacional de Ergonomia e Usabilidade de Interfaces Humano Tecnológica: Produto, Informações Ambientes Construídos e Transporte

$17^{\circ}$ USIHC - Congresso Internacional de Ergonomia e Usabilidade

de Interfaces Humano Computador

Quadro 9. Sistematização do Nono Artigo da Revisão Sistemática

\begin{tabular}{|c|c|}
\hline Ano & 2017 \\
\hline Título & Autismo e Conforto Ambiental. \\
\hline Autor & TRONCOSO, M.; CAVALCANTE, N. \\
\hline Objetivo & $\begin{array}{l}\text { Busca refletir sobre quais diretrizes devem orientar projetos de espaços ao ar } \\
\text { livre mais adaptados e inclusivos aos autistas. }\end{array}$ \\
\hline Métodos & Pesquisa Bibliográfica. \\
\hline Resultados & $\begin{array}{c}\text { A pesquisa mostrou com base no referencial teórico apresentado pelas autoras, } \\
\text { a importância em projetar espaços públicos em vista dos portadores do TEA, a } \\
\text { fim de promover maior qualidade a esses espaços e o desenvolvimento } \\
\text { cognitivo e sensorial desses indivíduos. }\end{array}$ \\
\hline Publicação & $16^{\circ}$ ERGODESING, USIHC, CINAHPA. \\
\hline Local & Brasil \\
\hline
\end{tabular}

Fonte: realizado pelos autores (2019)

Para análise deste último artigo as autoras declaram que, a linguagem arquitetônica lúdica e abstrata pode dialogar melhor com os autistas, no sentido de gerar uma empatia destes com o seu entorno espacial, e assim, tornando o mundo a sua volta mais adaptado, seguro, prazeroso e inclusivo. Entretanto, as autoras afirmam que, não existem ainda propostas específicas para os espaços ao ar livre adequado ao uso dos autistas.

As autoras da pesquisa, afirmam também que, as necessidades dos portadores do TEA envolvem questões de caráter sensorial, e por esse motivo, não seria errado admitir que as soluções buscadas para tais questões também estão relacionadas ao campo da ergonomia e do conforto ambiental. De acordo com o psicólogo Michael Shermer, as crianças autistas estão focadas na tarefa de organizar, ou completar uma sequência, e por essa razão, as mesmas usufruem de padrões racionais, repetitivos e contínuos, os quais contribuem para garantir determinada estabilidade com o seu entorno.

A característica de padrões repetitivos presente nos autistas pode ser considerada como um direcionamento nos projetos arquitetônicos ou até mesmo o reforço da ideia de continuidade em 
$17^{\circ}$ ERGODESIGN \& USIHC 2019

PUC-Rio, 11 a 13 de dezembro

Rio de Janeiro, RJ, Brasil $17^{\circ}$ Ergodesign - Congresso Internacional de Ergonomia e Usabilidade de Interfaces Humano Tecnológica: Produto, Informações Ambientes Construídos e Transporte

$17^{\circ}$ USIHC - Congresso Internacional de Ergonomia e Usabilidade de Interfaces Humano Computador

espaços circulares, e assim promovendo uma comunicação mais prazerosa destes usuários com o espaço construído.

Troconso e Cavalcante (2017), também destacam outros fatores importantes para o favorecimento da acessibilidade nos projetos de espaços públicos para os usuários, tais como: o uso do lúdico, do brincar, das formas abstratas e das figuras geométricas com foco em sequências repetidas.

Em vista disso, as autoras concluíram que, as orientações desenvolvidas por Mostafa (2008), podem contribuir para a elaboração de diretrizes para os espaços ao ar livre utilizado por autistas. E a partir daí, estabelecer suporte para às diretrizes de projeto de espaços públicos infantis. E nesse sentido, é importante pensar em soluções arquitetônicas e urbanísticas para tais espaços de vivência externa, a fim de minimizar os ruídos da cidade e promover a integração do portador do TEA.

Com base nas análises dos nove artigos descritos acima, tem-se a seguir, o Quadro 10, que tem por objetivo a síntese do contexto central do conteúdo discorrido nessa RSL.

Quadro 10. Síntese Geral dos Artigos da Revisão Sistemática

\begin{tabular}{|c|c|}
\hline \multicolumn{2}{|c|}{ SÍNTESE GERAL } \\
\hline ARTIGOS & CONTEXTO GERAL \\
\hline Architecture For Autism: Autism Aspects in School Design. & Diretrizes baseadas no Modelo da Teoria do Design Sensorial. \\
\hline $\begin{array}{l}\text { Autism-Friendly Architecture From The Outside In And The Inside Out: An } \\
\text { Explorative Study Based On Autobiographies Of Autistic People. }\end{array}$ & Análise comparativa: guia de design $\mathrm{x}$ autobiografias de autistas. \\
\hline Designing Schools for Students on the Spectrum & $\begin{array}{c}\text { Análise e descrição de estudos realizados com base em Diretrizes do Design } \\
\text { para portadores do TEA. }\end{array}$ \\
\hline $\begin{array}{l}\text { Exploring the impact of the design of the physical classroom environment on } \\
\text { young children with autism spectrum disorder (ASD) }\end{array}$ & $\begin{array}{l}\text { Revisão Sistemática: em vista das orientações e recomendações para } \\
\text { intervenções no ambiente construído educacional para autistas. }\end{array}$ \\
\hline $\begin{array}{c}\text { Sensory Spaces: Sensory Learning - An Experimental Approach To Educating } \\
\text { Future Designers To Design Autism Schools }\end{array}$ & Projeto Experimental realizado discentes em Design para estudantes autistas. \\
\hline $\begin{array}{c}\text { An Observational Study of Classroom Acoustical Design and Repetitive } \\
\text { Behaviors in Children With Autism }\end{array}$ & $\begin{array}{l}\text { Análise comparativa: comportamento repetitivo x níveis de ruído em um } \\
\text { ambiente educacional. }\end{array}$ \\
\hline Acessibilidade entre Mundos: uma Arquitetura mais Inclusiva aos Autistas & $\begin{array}{c}\text { Recomendações com base nas Diretrizes do Design e no American } \\
\text { Association on Mental Retardation. }\end{array}$ \\
\hline A Ergonomia e a Arquitetura no Cuidado das Pessoas com Transtorno Mental & $\begin{array}{l}\text { Elaboração de Mapa de Riscos (com base na NR 9, 1978) } \\
\text { para três estudos de caso com usuários autistas. }\end{array}$ \\
\hline Autismo e Conforto Ambiental & Diretrizes estabelecidas para espaços ao ar livre destinados aos autistas. \\
\hline
\end{tabular}




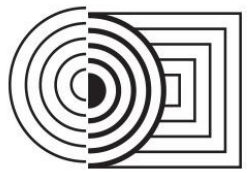

\section{$17^{\circ}$ ERGODESIGN \& USIHC 2019 \\ PUC-Rio, 11 a 13 de dezembro \\ Rio de Janeiro, RJ, Brasil}

$17^{\circ}$ Ergodesign - Congresso Internacional de Ergonomia e Usabilidade de Interfaces Humano Tecnológica: Produto, Informações Ambientes Construídos e Transporte

$17^{\circ}$ USIHC - Congresso Internacional de Ergonomia e Usabilidade

de Interfaces Humano Computador

Conforme a análise geral dos artigos foi visto que, os estudos (03 artigos - destacados pela cor de preenchimento: azul-claro), direcionaram as suas investigações com base na teoria aplicada pelo Design Sensorial, a qual foi apresentada nas pesquisas realizadas por Mostafa (2014). Desse modo, compreende-se que há uma referência sólida com relação às recomendações para projetos que visam às características dos autistas. Pois, nesses 3 trabalhos teóricos, os autores basearam-se nos conceitos abordados pela Teoria do Design Sensorial, e assim, desenvolveram as suas propostas de acordo com o contexto de suas respectivas pesquisas.

No entanto, as pesquisas (destacadas pela cor de preenchimento: amarelo-claro) são caracterizadas como pesquisas elaboradas a partir de casos reais, ou seja, estudos de casos aplicados a ambientes internos e distintos. Entretanto, dos 4 estudos de caso, 3 foram dirigidos para espaços educacionais, porém, todas as experiências foram executadas com usuários autistas.

O artigo destacado pela cor de preenchimento verde-claro é diferenciado por se tratar de um trabalho teórico singular, no qual foi baseado nos conceitos teóricos do Design e de autobiografias de portadores do TEA. A análise comparativa é significativa, devido ao destaque aportado sobre as interpretações espaciais desses indivíduos.

Por fim, o artigo destacado pela cor de preenchimento magenta-claro, evidencia uma Revisão Sistemática, na qual concentra reflexões fundamentais sobre a importância do Design para o ambiente educacional para pessoas portadoras do autismo.

\section{CONSIDERAÇÕES FINAIS}

Desse modo, conforme a análise realizada nesta Revisão Sistemática (RS) foi visto que, os ambientes construídos através de diretrizes e recomendações do design e da arquitetura podem promover segurança, conforto, qualidade e significados aos portadores do TEA. Uma vez que, as suas interpretações sensoriais com relação ao entorno são fundamentais e que precisam ser reconhecidas, para que assim, o ambiente seja mais adequado e almejado as suas vontades, necessidades e desejos.

Além disso, é importante reconhecer também que, as pesquisas sobre esse cenário precisam de um aumento em suas investigações. Uma vez que, as pesquisas sobre outros ambientes construídos para autistas foram praticamente escassas. Em contrapartida as pesquisas nas áreas de educação e saúde mostram-se muito mais em evidência.

\section{REFERÊNCIAS}

In: ARCHITECTS AND BUILDING BRANCH, DEPARTMENT FOR EDUCATION. Area guidelines for SEND and alternative provision Including special schools, alternative provision, specially resourced provision and units, Building Bulletin. London: HM Government. 2015.

BARBOSA, M. O.; FUMES, N. L. F., Pontos e contrapontos no universo do atendimento educacional especializado para educandos com transtorno do espectro autista, Revista Ibero-Americana de Estudos em Educação 1, no 11 (2016): 467-77 


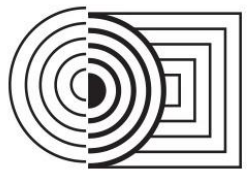

BAIO, J. et al. Prevalence of Autism Spectrum Disorder Among Children Aged 8 Years Autism and Developmental Disabilities Monitoring Network, 11 Sites, United States, 2014. Surveillance Summaries, [s. l.], abr., 2018. Disponível em: https://www.cdc.gov/mmwr/volumes/67/ss/ss6706a1.htm. Acesso em: 15 mai. 2019.

BAKER, A., LANE, A., ANGLEY, M., \& YOUNG, R. The relationship between sensory processing patterns and behavioural responsiveness in autistic disorder:

A pilot study. Journal of Autism and Developmental Disorders, 2008. doi:10.1007/s10803-0070459-0

BARANEK, G., FOSTER, L., \& BERKSON, G. Tactile defensiveness and stereotyped

behaviors. The American Journal of Occupational Therapy, 1997. DOI:10.5014/ajot.51.2.91

BRAND, A. Living in the community. London: Helen Hamlyn Centre, Royal College of Art. 2010.

VASCONCELOS, M. C.; FALCÃO, C. S. A Ergonomia e a Arquitetura no Cuidado das Pessoas com Transtorno Mental, NO 1 (S.D.). 2018.

DELACATO, C. H. The ultimate stranger. Novato: Academic Therapy Publications. 1974.

DELMOLINO, L. \& HARRIS, S. L. Matching children on the autism spectrum to classrooms: A guide for parents and professionals. Journal of Autism and Developmental Disorders, 2012. doi: 10.1007/s10803-011-1298-6

GRANDIN, T. Thinking in pictures and other reports from my life with autism. New York: Doubleday. 1995.

GRANDIN, T. Sensory therapies and autism. 2012. Disponível em: http://www.templegrandin.com. Acesso em 15 de junho de 2019.

HOWE, F., \& STAGG, S. How sensory experiences affect adolescents with an autistic spectrum condition within the classroom. Journal of Autism and Developmental Disorders, 2016. doi:10.1111/1471-3802.12092. 2016.

KANAKRI, S. M.; SHEPLEY, M.; TASSINARY, L. G.; VARNI, J. W.; FAWAZ, H. M. An Observational Study of Classroom Acoustical Design and Repetitive Behaviors in Children With Autism. Journal Environment and Behavior. Reino Unido. 2016.

KINNAER, M.; BAUMERSI, S.; HEYLIGHEN, A. Autism-friendly architecture from the outside in and the inside out: an explorative study based on autobiographies of autistic people. Springer Science Business, Alemanha, 2015.

LOVE, J. Sensory Spaces: Sensory Learning - An Experimental Approach to Educating Future Designers to Design Autism Schools. International Journal of Architectural Research, Reino Unido, 2018.

MCALLISTER, K. The ASD friendly classroom-design complexity, challenge \& characteristics. In Z Michalewicz (Ed.), Paper presented at Design Research Society International Conference. Canada. 2010.

MARTIN, C. S. Exploring the Impact of the Design of the Physical Classroom Environment on young children with Autism Spectrum Disorder (ASD). Journal of Research in Special Educational Needs, EUA, 2014. 
MATIN SADR N., HAGHGOO H., SAMADI S. A., RASSAFIANI M., BAKHSHI E., HASSANABADI $\mathrm{H}$. The Impact of Dynamic Seating on Classroom Behavior of Students with Autism Spectrum Disorder. Iranian Journal of Child Neurology, Iran, 2017.

MOSTAFA, M. An architecture for Autism. Archnet-IJAR, 2(1), 2007.

MOSTAFA, M. An architecture for autism: Concepts of design intervention for

the autistic user. International Journal of Architectural Research. 2008.

MOSTAFA, M. Architecture for autism: autism aspectss in school design. International Journal of Architectural Research. [s. I.] Mar. 2014. DOI: 10.26687/archnet-ijar.v8i1.314. Disponível em: https://archnet.org/publications/9101. Acesso em: 19 jun. 2019.

SCOTT, I. Designing learning spaces for children on the autistic spectrum. Good Autism Practice, 2009.

TRONCOSO M.; CAVALCANTE N. Autismo e Conforto Ambiental Autism and Environmental Comfort, XVI Congresso Internacional de Ergonomia Usabilidade de Interfaces Humano Tecnológica: Produto, Informações Ambientes Construídos e Transporte. ERGODESIGN, USIHC, CINAHPA. 2017.

TUFVESSON, C., \& TUFVESSON, J. The building process as a tool towards an allinclusive school: A Swedish example focusing on children with defined concentration difficulties such as ADHD, autism and Down's syndrome. Journal of Housing and the Built Environment, 2009. doi:10.1007/s10901-008-9129-6

VERGARA, L. G. L; TRONCOSO, M. U.; RODRIGUES, G. V. ACESSIBILIDADE ENTRE MUNDOS: uma arquitetura mais inclusiva aos autistas. no 1 (s.d.). 2018.

ULRIKE, A.L. Designing Schools for Students on the Spectrum. The Design Journal. An International Journal for All Aspects of Design, England, 2017.

WORLD HEALTH ORGANIZATION. Training for health care providers: Children and noise. 2016. Disponível em: www.who.int/ceh. Acesso em 20 de junho de 2019.

\section{AGRADECIMENTOS}

Os autores deste artigo agradecem a CAPES e ao Programa de Pós Graduação em Design da UFPE pela contribuição recebida para o desenvolvimento deste trabalho científico. 